\title{
The Low Income Diet and Nutrition Survey: implications for relationships between diet and disease
}

\author{
J. Cade \\ Nutritional Epidemiology Group, 30-32 Hyde Terrace, University of Leeds, Leeds LS2 9LN, UK
}

The Low Income Diet and Nutrition Survey (LIDNS) ${ }^{(1)}$ makes it possible to address key diet-related issues of public health importance in the poorest $15 \%$ of the population in terms of material deprivation. Results have shown a similar, but perhaps more extreme, picture in terms of diet and lifestyle to that of the general population. When considering implications for this population in terms of diet and disease it is important to place the diet in context with other health-related factors. This population had high smoking rates, particularly amongst younger adults. There were very low levels of regular moderate or vigorous activity. Of the subjects $>16$ years $53 \%$ had a pre-existing illness, in $40 \%$ of whom this illness limited their activity levels.

In general, average consumption of fruit and vegetables were approximately half the recommended five portions per $\mathrm{d}^{(2)}$. One-third of men, women and boys consumed no fruit in the $24 \mathrm{~h}$ recall. NSP consumption was low. Use of fat spreads and oils, soft drinks, red and processed meats, whole milk and table sugar were higher than those of the general population. These dietary patterns contribute to health inequalities found in low-income populations, including high rates of obesity. Of the adults $>60 \%$ and $>30 \%$ of children were overweight or obese. In addition, central obesity was higher in this population than in the general population. These diet and associated lifestyle habits may also result in higher morbidity from CVD, certain cancers (potentially colon and breast cancers) and maternal and child health considerations including breast-feeding and family diet practices. In addition, high levels of sugar intake may have resulted in poorer dental health than that found in the general population.

The diets in younger adults and children appeared to be poorer than those for older adults. If these patterns continue, future rates of obesity and chronic disease may increase. There is a desire in this population to improve diet but price considerations affected ability to change.

LIDNS was funded by the Food Standards Agency and conducted by the National Centre for Social Research with King's College London and University College London.

1. Nelson M, Erens B, Bates B, Church S \& Boshier T (editors) (2007) Low Income Diet and Nutrition Survey. London: The Stationery Office.

2. Department of Health (2000) The NHS Plan. London: Department of Health. 